\title{
Overexpression of Aurora-A promotes laryngeal cancer progression by enhancing invasive ability and chromosomal instability
}

\author{
Hao Zhang $\cdot$ Xuehua Chen $\cdot$ Yuesheng Jin $\cdot$ \\ Bingya Liu $\cdot$ Liang Zhou
}

Received: 13 February 2011 / Accepted: 2 May 2011 / Published online: 17 May 2011

(C) The Author(s) 2011. This article is published with open access at Springerlink.com

\begin{abstract}
The purpose of the study was to investigate the expression of Aurora-A in human laryngeal squamous cell carcinoma (LSCC) and to explore the effects of Aurora-A silencing on invasion and chromosomal instability in laryngeal cancer HEp-2 cells. The expression of Aurora-A mRNA and protein were studied using reverse transcription-PCR and Western blot in LSCC tissues and corresponding normal epithelium, respectively. In addition, the correlation between Aurora-A expression and clinicopathologic characteristics was analyzed in LSCC patients. Furthermore, HEp-2 cells were transfected with Aurora-A short hairpin RNA and the effects of knockdown of AuroraA on tumor invasion and chromosomal instability were investigated. The results showed that expression of AuroraA mRNA was significantly upregulated in laryngeal tumor tissue compared with that in normal tissue $(P=0.001)$, and overexpression of Aurora-A was found in $64.0 \%$ (16 of 25) of the patients by Western blotting. Upregulation of Aurora-A mRNA was significantly correlated with regional lymph node metastasis $(P=0.007)$ and clinical stage III/IV $(P=0.022)$. Overexpression of Aurora-A was significantly associated with lymph node metastasis $(P=0.027)$. Furthermore, disruption of Aurora-A using RNA interference
\end{abstract}

\footnotetext{
H. Zhang $\cdot$ L. Zhou ( $ه)$

Department of Otolaryngology, Head and Neck Surgery,

Eye Ear Nose and Throat Hospital, Fudan University,

83 FenYang Road, Shanghai 200031, China

e-mail: zhoulent@126.com

X. Chen · B. Liu

Department of Surgery, Ruijin Hospital, School of Medicine,

Jiao Tong University, Shanghai, China

Y. Jin

Department of Clinical Genetics,

University Hospital, Lund, Sweden
}

technique suppressed invasive ability and chromosomal instability in HEp-2 cells. In conclusion, Aurora-A expression is elevated in human LSCC and associated with regional lymph node metastasis and late clinical stage. Overexpression of Aurora-A may contribute to LSCC carcinogenesis and progression partially due to enhancement of invasion ability and chromosomal instability.

Keywords Aurora kinase · Laryngeal neoplasms . Cell invasion Chromosomal instability

\section{Introduction}

Laryngeal squamous cell carcinoma (LSCC) is a world-wide malignancy and its survival rate is not improving. Since larynx has a crucial physiological function in respiration and phonation, LSCC has a severe impact on the quality of life of patients [1]. Despite recent advances in treatments, the invasion and metastasis are still considered as the principal reasons of mortality of LSCC patients. Currently the therapeutic strategies included surgical intervention, radiotherapy, and chemotherapy, either alone or in combination. However, they are less effective in advanced cases. Therefore, identifying new marker as tumor staging and new strategy for the treatment of LSCC is in urgent demand.

Tumorigenesis is a multistep accumulation of genetic alterations, driving indefinite proliferation and malignant transformation of normal human cells. The cellular mitoses are controlled by several serine/threonine kinases, known as mitotic kinases. Aurora-A is a member of the Aurora/ Ipl1p family of serine/threonine kinases, which is implicated in concerted progression of each mitotic event. Aurora-A amplification was detected in breast, ovarian, colon, prostate, neuroblastoma and cervical cancer cell 
lines. Ectopic expression of Aurora-A in mouse NIH 3T3 cells and human breast epithelial cells led to centrosome amplification and cell transformation in vitro [2]. Furthermore, a correlation between overexpression of Aurora-A and tumor development has been described in colorectal cancer and cervical cancer [3, 4]. These findings suggest that Aurora-A is a pivotal kinase whose overexpression leads to carcinogenesis in mammalian cells.

The expression of Aurora-A both in mRNA and protein level in a series of LSCC patients was rarely reported. In addition, the correlation between expression of Aurora-A and clinical aggressiveness in cancers remains controversial. Furthermore, the effects Aurora-A exerts on tumor invasion and chromosomal instability in laryngeal HEp- 2 cells are not well investigated. In this study, we examined the expression of Aurora-A and its association with clinicopathologic features in LSCC patients. We also delivered a specifically designed short hairpin RNA (shRNA) targeting Aurora-A into laryngeal cancer HEp-2 cells, to evaluate the inhibitory effect of Aurora-A knockdown on cell invasion ability and chromosomal instability status in HEp-2 cells. The results showed that Aurora-A was overexpressed in LSCC and Aurora-A silencing by siRNA could effectively inhibit the invasion and chromosomal instability in HEp-2 cells.

\section{Methods}

Patients and clinical tissue samples

All tissues were obtained and kept in liquid nitrogen from patients who were diagnosed with primary LSCC and underwent a surgical procedure in the Department of Otolaryngology-Head and Neck Surgery, Eye Ear Nose and Throat Hospital, Fudan University from April to July in 2009. Written informed consents were obtained from the patients regarding the surgical operation and the use of resected specimens for research. All tumors were confirmed by the Department of Pathology of the hospital and were classified according to the 3rd edition of TNM staging of head and neck cancer [5]. Non-neoplastic squamous epithelium which was at least $1 \mathrm{~cm}$ from the tumor margin was used as paired normal adjacent tissues. Patients' clinical data were retrieved from medical records, including gender, age, tumor site, tumor and lymph node classification, histological grade and tumor stage. In addition, patients with preoperative chemotherapy and radiation were excluded.

Extraction of tissue RNA and quantitative reverse transcription-PCR

Total RNA was extracted from the frozen stored tissues of patients using the TRIzol reagent (Invitrogen, CA) according to the manufacturer's protocol. $2 \mu \mathrm{g}$ of total RNA were reverse transcribed to cDNA using the Reverse Transcription System (Promega, WI, USA). cDNA sequence were then amplified by quantitative PCR with SYBR Green (Invitrogen, CA). In brief, $1 \mu \mathrm{l}$ of cDNA was added in a $20 \mu 1$ reaction mixture containing $2 \times$ SYBR Green PCR Master Mix (Applied Biosystem, CA). The PCR primers used for Aurora-A were $5^{\prime}$ ACT GAC CAC CCA AAA TCT GC-3' for the forward primer and 5'-TGG AAT ATG CAC CAC TTG GA-3' for the reverse primer, For glyceraldehyde-3-phosphate dehydrogenase (GAPDH), the forward primer was $5^{\prime}-\mathrm{G}$ GA CCT GAC CTG CCG TCT AG-3', and the reverse primer was $5^{\prime}$-GTA GCC CAG GAT GCC CTT GA-3'. PCR conditions were $50^{\circ} \mathrm{C}$ for $2 \mathrm{~min}$, and $95^{\circ} \mathrm{C}$ for $10 \mathrm{~min}$, followed by 40 cycles at $95^{\circ} \mathrm{C}$ for $15 \mathrm{~s}, 59^{\circ} \mathrm{C}$ for $60 \mathrm{~s}$. The expression level of Aurora-A mRNA was internally normalized using GAPDH. Aurora-A mRNA level of tumor or normal tissue sample was represented as $2^{-\Delta \mathrm{Ct}}$, in which the $\Delta \mathrm{Ct}$ represents difference between thresholds of Aurora-A and GAPDH $\left(\mathrm{Ct}_{\text {Aurora-A }}-\mathrm{Ct}_{\text {GAPDH }}\right)$.

Protein extraction and Western blotting assay

Frozen tissue samples were ground to a fine powder using a mortar and pestle under liquid nitrogen. Tissues were then dissolved in T-PER Tissue Protein Extraction Reagent (Thermo Fisher Scientific Inc., IL) on ice for at least $30 \mathrm{~min}$ and centrifuged at $14,000 \mathrm{~g}$ for $15 \mathrm{~min}$ at $4^{\circ} \mathrm{C}$. Protein concentration was determined using the BCA Protein Assay Reagent (Thermo Fisher Scientific Inc., IL). $30 \mu \mathrm{g}$ of protein samples were loaded onto a $10 \%$ SDS-PAGE. After electrophoresis, the separated proteins were transferred to a PVDF membrane (BioRad, CA) overnight at $4^{\circ} \mathrm{C}$. Membrane was blotted with 5\% non-fat dry milk dissolved in $1 \times$ TBS for $2 \mathrm{~h}$ in room temperature, and then incubated in 1:2,000 primary anti-Aurora-A rabbit polyclonal antibody (Abcam, UK) overnight. After washing with TBST and incubation with horseradish peroxidase-conjugated antirabbit secondary antibody (Abcam, UK) for $1 \mathrm{~h}$ diluted in 1:3,000, the protein bands were detected using Immobilon Western chemiluminescent HRP substrate (Millipore, MA). The membrane was stripped with $0.2 \mathrm{M} \mathrm{NaOH}$ and re-probed using GAPDH antibody diluted in 1:3,000 (Abcam, UK). The band intensity of Aurora-A and GAPDH were quantified by AlphaEaseFC software (Alpha Innotech, CA) and Aurora-A expression of each specimen was determined by the ratio of Aurora-A and GAPDH intensity. Afterward, for a specific clinical case the Aurora-A expression was calculated as $\mathrm{T} / \mathrm{N}$ ratio, i.e., Aurora-A in tumor/Aurora-A in corresponding normal tissue. 


\section{Cell cultures and shRNA transfection}

Human laryngeal cancer HEp-2 cell line was grown in RPMI1640 (Invitrogen, CA) supplemented with $10 \%$ fetal bovine serum and $200 \mu \mathrm{g} / \mathrm{ml}$ penicillin at $37^{\circ} \mathrm{C}$ under $5 \% \mathrm{CO}_{2}$. Cells were re-fed with fresh medium every $2-3$ days. A chemically synthesized oligonucleotide encoding Aurora-A (NM_003600) short hairpin RNA was inserted downstream of U6 promoter of the pGC-silencer-U6/GFP/ Neo plasmid (pGC-shAurora-A) and cloned (Genchem, China). The sequence of the oligonucleotide targeting Aurora-A was 5'-CAAAGAATCAGCTAGCAAA-3'. A non-specific stem-loop DNA vector (pGC-shNS) was also generated for use as a negative control. HEp-2 cells were transfected with pGC-shAurora-A or pGC-shNS with Lipofectin reagent (Invitrogen, CA) according to the manufacturer's instructions. Briefly, HEp-2 cells $\left(4 \times 10^{5}\right)$ were plated into individual wells of six-well dish. $24 \mathrm{~h}$ later, $4 \mu \mathrm{g}$ of plasmid and $10 \mu \mathrm{l}$ of Lipofectamine (Invitrogen, CA) were added to $250 \mu \mathrm{l}$ of Opti-MEM (Invitrogen, CA), respectively, and incubated for $5 \mathrm{~min}$ at room temperature. Afterward, the plasmid and Lipofectamine solutions were mixed gently and placed for $20 \mathrm{~min}$. Then the mixture was diluted with $2 \mathrm{ml}$ RPMI with $10 \% \mathrm{FBS}$ and added to the well at $37^{\circ} \mathrm{C}$ under $5 \% \mathrm{CO}_{2}$ for $24 \mathrm{~h}$. Subsequently, the cells were selected against G418 at a final concentration of $800 \mu \mathrm{g} / \mathrm{ml}$ for 3 weeks and stable Aurora-A knockout HEp2 cells were acquired.

Trans-well assay

Cell invasion was investigated with chemotaxis chambers (Neuro Probe, Inc. Gaithersburg, MD). Cells $\left(1 \times 10^{5}\right)$ in $200 \mu \mathrm{l}$ of RPMI1640 were added to the upper chamber and $500 \mu \mathrm{l}$ of RPMI1640 with 10\%FBS was filled in the lower chamber. A polycarbonate membrane with $8 \mu \mathrm{m}$-diameter pores was placed between the two chambers. The cells were allowed to migrate at $37^{\circ} \mathrm{C}$ in a $5 \% \mathrm{CO}_{2}$ humidified incubator. $24 \mathrm{~h}$ later, non-migrating cells on the upper chamber were removed with a cotton swab. The filter membrane were then fixed in methanol for $10 \mathrm{~min}$ and stained with Giemsa solution for $20 \mathrm{~min}$. Migrated cells on the membrane were counted under a microscope. Invasion was quantified by counting the migrated cells in three random fields per filter.

\section{Chromosome segregation analysis}

For analysis of chromosomal instability events, cells on chamber slides were washed with PBS, fixed in methanol:acetic acid (3:1) at $-20^{\circ} \mathrm{C}$ for $30 \mathrm{~min}$, air dried, and stained with hematoxylin and eosin. Anaphase cells showing at least one string of chromatin connecting the poles were classified as harboring an anaphase bridge. Anaphase cells with a chromosome configuration showing three or more spindle poles were classified as multipolar mitoses. At least 100 anaphase and metaphase cells were analyzed in each group.

Statistical analysis

Aurora-A mRNA level between tumor and normal groups was compared by independent-samples $t$ test with a Statistical Product and Service Solutions software (SPSS, Inc., Chicago, IL). The association of tumor Aurora-A mRNA and clinicopathologic characteristics was analyzed by $t$ test in two classes or Student-Newman-Keuls test between various groups. The relationship between Aurora-A protein expression and clinicopathologic characteristics was analyzed by Fisher's exact test in two groups and Pearson's Chi-square test in more than two groups. For cellular experiments, statistical differences among groups were evaluated using one-way ANOVA analysis. Statistical significance was considered at the value of $P<0.05$.

\section{Results}

Aurora-A mRNA upregulation and protein overexpression in LSCC

To investigate whether Aurora-A abnormalities are related to human LSCC, we first analyzed expression level of Aurora-A mRNA in clinical samples. Quantitative reverse transcriptase-PCR was used in 37 tumor specimens and their paired normal adjacent tissues. The mean expression of Aurora-A mRNA was $0.0916 \pm 0.0924$ (range $2.21 \times 10^{-8}$ to 0.289$)$ in tumor samples, and 0.0255 \pm 0.0794 (range $4.11 \times 10^{-8}$ to 0.298 ) in normal adjacent tissues. Aurora-A mRNA expression level in LSCC was significantly higher than that of normal epithelium $(P=0.001$, Fig. 1a).

We then examined the Aurora-A protein expression in tumor samples and their paired normal adjacent tissues. 25 patients' paired samples were successfully analyzed by Western blotting. We defined the cases with a Aurora-A protein $\mathrm{T} / \mathrm{N}$ ratio over 1.20 as overexpression, whereas the cases with a $\mathrm{T} / \mathrm{N}$ ratio less than 1.20 were classified as nonoverexpression. Aurora-A overexpression was detected in 16 of 25 (64.0\%) LSCC cases (Fig. 1b). The correlation between Aurora-A overexpression and mRNA upregulation was not found. We also examined expression of Aurora-A protein in human LSCC HEp-2 cells. As shown in Fig. 1c, HEp-2 cells revealed high expression of Aurora-A level as HeLa cell did. 


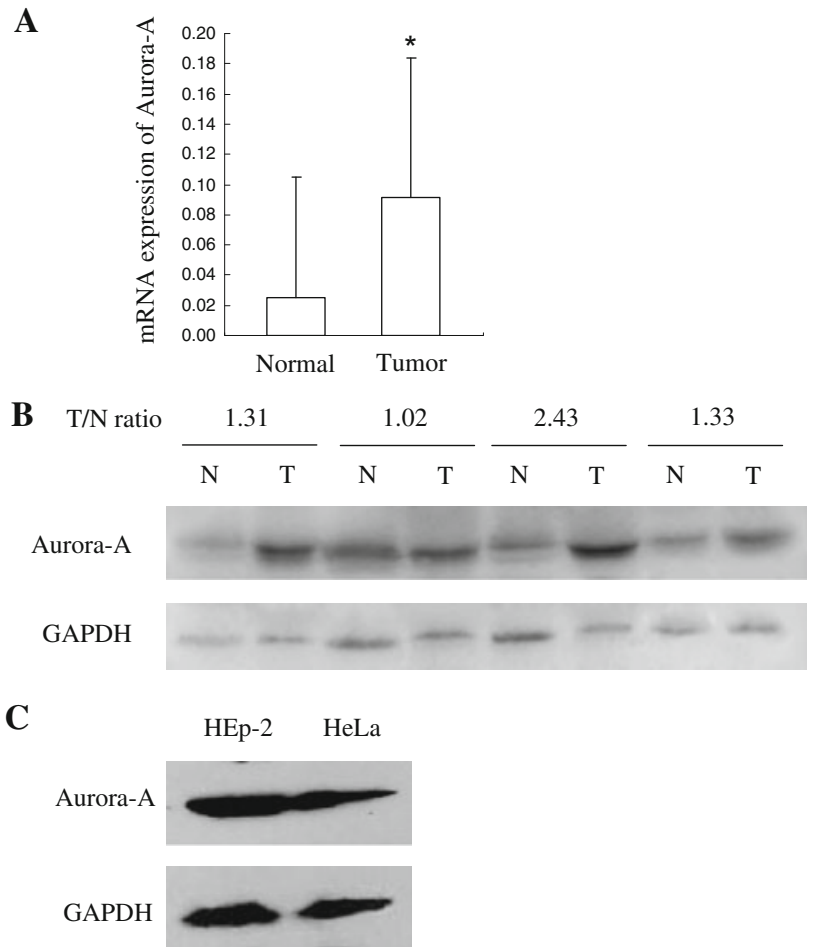

Fig. 1 Expression of Aurora-A in 37 LSCC patients and HEp-2 cells. a mRNA level of Aurora-A in tumor tissues and corresponding normal adjacent tissues was analyzed by quantitative reverse transcriptase-PCR. Aurora-A mRNA level in LSCC was significantly higher than that of normal epithelium $(* P=0.001)$. b Protein expression of Aurora-A in LSCC samples $(T)$ and their corresponding normal epithelium $(N)$ were investigated by Western blot. Aurora-A expression in each patient was evaluated using $\mathrm{T} / \mathrm{N}$ ratio (the ratio of Aurora-A in tumor/Aurora-A in corresponding normal tissue). $\mathbf{c}$ Protein expression of Aurora-A in HEp-2 cells. HeLa cells were used as positive control

Overexpression of Aurora-A is correlated with regional lymph node metastasis

The correlation between Aurora-A expression and patients clinicopathological characteristics was examined. As summarized in Table 1, Aurora-A mRNA upregulation was correlated with regional lymph node metastasis $(P=0.007)$ and clinical stage III/IV $(P=0.022)$. There was no significant correlation between Aurora-A mRNA and patient's gender $(P=0.788)$, age $(P=0.305)$, tumor anatomy site $(P=0.905)$, T category $(P=0.582)$, as well as pathological grade $(P=0.814)$.

Aurora-A protein overexpression $(\mathrm{T} / \mathrm{N}$ ratio $>1.20)$ was significantly associated with the occurrence of regional lymph node $(P=0.027$, Table 2$)$. Correlation between Aurora-A overexpression with patient's gender $(P=0.360)$, age $(P=0.411)$, tumor site $(P=0.172)$, T category $(P=0.171)$, histological grade $(P=0.396)$ and clinical stage III/IV $(P=0.097)$ were not found.
Table 1 Correlation between Aurora-A mRNA level and patients clinicopathologic characteristics

\begin{tabular}{|c|c|c|c|c|}
\hline $\begin{array}{l}\text { Patients } \\
\text { characteristics }\end{array}$ & $\begin{array}{l}\text { Case } \\
\text { number }\end{array}$ & $\begin{array}{l}\text { Aurora-A } \\
\text { mRNA level } \\
\text { (mean) }\end{array}$ & SD & $P$ value \\
\hline \multicolumn{5}{|l|}{ Gender } \\
\hline Male & 36 & 0.0910 & 0.0935 & 0.788 \\
\hline Female & 1 & 0.1166 & & \\
\hline \multicolumn{5}{|l|}{ Age } \\
\hline$\leq 64$ & 23 & 0.1040 & 0.0905 & 0.305 \\
\hline$\geq 65$ & 14 & 0.0714 & 0.0950 & \\
\hline \multicolumn{5}{|l|}{ Tumor site } \\
\hline Supraglottic & 17 & 0.0853 & 0.0710 & 0.905 \\
\hline Glottic & 13 & 0.1009 & 0.1082 & \\
\hline Subglottic & 7 & 0.0899 & 0.1182 & \\
\hline \multicolumn{5}{|l|}{$\mathrm{T}$ category } \\
\hline $\mathrm{T} 1$ & 6 & 0.0592 & 0.0485 & 0.582 \\
\hline $\mathrm{T} 2$ & 22 & 0.0983 & 0.0960 & \\
\hline $\mathrm{T} 3$ & 8 & 0.1087 & 0.1094 & \\
\hline $\mathrm{T} 4$ & 1 & 0.0035 & & \\
\hline \multicolumn{5}{|c|}{ Lymph node metastasis } \\
\hline $\mathrm{N}(-)$ & 23 & 0.0607 & 0.0737 & $0.007^{\mathrm{a}}$ \\
\hline $\mathrm{N}(+)$ & 14 & 0.1425 & 0.0997 & \\
\hline \multicolumn{5}{|c|}{ Histologic grade } \\
\hline 1 & 5 & 0.0669 & 0.0768 & 0.814 \\
\hline 2 & 30 & 0.0961 & 0.0961 & \\
\hline 3 & 2 & 0.0872 & 0.1075 & \\
\hline \multicolumn{5}{|l|}{ Clinical stage } \\
\hline I, II & 19 & 0.0584 & 0.0739 & $0.022^{\mathrm{a}}$ \\
\hline III, IV & 18 & 0.1268 & 0.0985 & \\
\hline
\end{tabular}

a Independent-samples $t$ test

Suppression of Aurora-A inhibits HEp-2 cell invasion

As we found Aurora-A overexpression was correlated with regional metastasis in clinical data, we further examined the role of Aruora-A in HEp-2 cell invasion. Aurora-A shRNA plasmid was constructed and transfected to HEp-2 cells to knockdown endogenous Aurora-A. Aurora-A protein was shown to be reduced after transfection (Fig. 2a). HEp-2/ Knockdown ratio for Aurora A protein was 2.31, exceeding the threshold for $\mathrm{T} / \mathrm{N}$ ratio we determined for clinical specimens. In contrast, a non-specific shRNA was used as negative control and showed no Aurora-A suppression in the experiment. With suppression of Aurora-A, cell invasion capability was inhibited by $50 \%$ in Aurora-A shRNA transfected HEp-2 cells compared with non-specific shRNA transfected cells shown in Trans-well assay $(P<0.01$, Fig. 2b, c, d, e). Therefore, suppression of Aurora-A led to inhibition of HEp-2 cell invasion. 
Table 2 The relationship between Aurora-A protein expression and patients clinicopathologic characteristics

\begin{tabular}{|c|c|c|c|}
\hline $\begin{array}{l}\text { Patient } \\
\text { characteristics }\end{array}$ & $\begin{array}{l}\text { Non-overexpression } \\
(\mathrm{T} / \mathrm{A} \text { ratio }<1.2)\end{array}$ & $\begin{array}{l}\text { Overexpression } \\
(\mathrm{T} / \mathrm{A} \text { ratio } \geq 1.2)\end{array}$ & $P$ value \\
\hline \multicolumn{4}{|l|}{ Gender } \\
\hline Male & 8 & 16 & \multirow[t]{2}{*}{0.360} \\
\hline Female & 1 & 0 & \\
\hline \multicolumn{4}{|l|}{ Age } \\
\hline$\leq 64$ & 6 & 7 & \multirow[t]{2}{*}{0.411} \\
\hline$\geq 65$ & 3 & 9 & \\
\hline \multicolumn{4}{|l|}{ Tumor site } \\
\hline Supraglottic & 4 & 5 & \multirow[t]{3}{*}{0.172} \\
\hline Glottic & 5 & 6 & \\
\hline Subglottic & 0 & 5 & \\
\hline \multicolumn{4}{|l|}{$\mathrm{T}$ category } \\
\hline $\mathrm{T} 1$ & 2 & 1 & \multirow[t]{4}{*}{0.171} \\
\hline $\mathrm{T} 2$ & 7 & 9 & \\
\hline $\mathrm{T} 3$ & 0 & 5 & \\
\hline $\mathrm{T} 4$ & 0 & 1 & \\
\hline \multicolumn{4}{|c|}{ Lymph node metastasis } \\
\hline $\mathrm{N}(-)$ & 9 & 9 & \multirow[t]{2}{*}{$0.027^{\mathrm{a}}$} \\
\hline $\mathrm{N}(+)$ & 0 & 7 & \\
\hline \multicolumn{4}{|l|}{ Histologic grade } \\
\hline 1 & 2 & 1 & \multirow[t]{3}{*}{0.396} \\
\hline 2 & 7 & 14 & \\
\hline 3 & 0 & 1 & \\
\hline \multicolumn{4}{|l|}{ Clinical stage } \\
\hline I, II & 7 & 6 & \multirow[t]{2}{*}{0.097} \\
\hline III, IV & 2 & 10 & \\
\hline
\end{tabular}

${ }^{\text {a }}$ Fisher's exact test

Knockdown of Aurora-A reduces abnormalities of chromosome segregation in HEp-2 cells

Aurora-A is an important regulator of bipolar spindle assembly and therefore essential for accurate chromosome segregation. To evaluate the effect of Aurora-A in chromosome segregation in HEp-2 cells, cell division morphology, demonstrated by anaphase bridges and multipolar mitoses, was analyzed. In Aurora-A shRNA transfected cells, anaphase bridges only occurred in $6.3 \pm 1.5 \%$ of anaphase cells, whereas anaphase bridges were present in $11.0 \pm 2.6 \%$ of non-specific shRNA transfected cells $(P<0.01$, Fig. 3a, b, c). Multipolar configurations were also less frequently observed in AuroraA shRNA transfected cells than control cells $(3.3 \pm 0.6 \%$ vs. $6.7 \pm 0.6 \%, P<0.01$, Fig. 3 a, d). These findings suggest that knockdown of Aurora-A reduced abnormal chromosome segregation.

\section{Discussion}

In the present study, we examined the expression of Aurora-A in human LSCC at mRNA and protein levels. The results showed that expression of Aurora-A mRNA and protein was significantly increased in laryngeal cancers compared with the most of the normal adjacent tissues. Likewise, expression of Aurora-A was also high in laryngeal cancer line HEp-2 cells. Furthermore, upregulation of Aurora-A mRNA was closely related to regional lymph node metastasis and tumor stage. These data indicated that alterations of Aurora-A at mRNA and protein levels are common abnormality in LSCC and may play important roles in LSCC progression.

Aurora-A is a mitotic-regulating serine/threonine kinase and its overexpression was associated with various cancers, including our series of laryngeal cancer patients. There are a range of mechanisms by which Aurora-A overexpression might promote tumor progression. Activation of Aurora-A transcription or supression of protein degradation may cause Aurora-A overexpression, resulting in tetraploid cells with multiple centrosomes [6]. These tetraploid cells would continue their division under inactivated p53-RB pathway and impaired mitotic G1 checkpoint. In addition, overexpression of Aurora-A might interact with other cellular proteins, such as PLK1, TPX2 and BRCA2, and induce abnormal spindle formation and promote mitotic entry through altering their physiological functions during mitotic process [7-9]. The panorama of these signaling pathways that Aurora-A involved in tumor progression needs further investigation.

However, we found that high expression of Aurora-A protein was not related to mRNA upregulation in the present study. Similar discrepancies between amplification and overexpression rates were also reported in hepatocellular carcinomas. Previous study found that amplification of Aurora-A was detected in only $3 \%$ of cases, but more than $60 \%$ of cases overexpressed Aurora-A protein in hepatocellular carcinomas [10]. This discrepancy may be accounted for that constitutive phosphorylation on Ser51 of Aurora-A induces protein stabilization and consequent accumulation in cancer cells, and exhibit overexpression of Aurora-A protein [11]. Therefore, suppression of protein degradation might be important for Aurora-A overexpression and its oncogenic role. Thus, it might be speculated that the inhibition of Aurora-A phosphorylation represents a novel way to decrease Aurora-A levels in cancer therapy.

In the aspect of cytogenetic mechanisms of tumorigenesis, it was well established that chromosomal instability might create structural and numerical chromosome aberrations [12]. The effect of Aurora-A overexpression on chromosomal instability to the progression of laryngeal cancer cells is rarely studied. In the present study, chromosomal 
Fig. 2 Transfection of AuroraA shRNA and Trans-well assay in HEp-2 cells. a Expression of Aurora-A was suppressed in HEp-2 cells transfected with Aurora-A shRNA detected by Western blot. GAPDH was used as internal control. A representative result of three independent experiments was showed. b Invasion of untransfected HEp-2 cells in Trans-well assay $(\times 100)$. $\mathbf{c}$ Invasion of nonspecific shRNA transfected HEp-2 cells $(\times 100)$. d Invasion of Aurora-A shRNA transfected HEp- 2 cells $(\times 100)$. e The number of migrated HEp- 2 cells transfected with Aurora-A shRNA was significantly less than those of untransfected cells. The number was expressed as mean \pm SD from three independent experiments. $(* P<0.01$, Student-Newman-Keuls test)

\section{A}

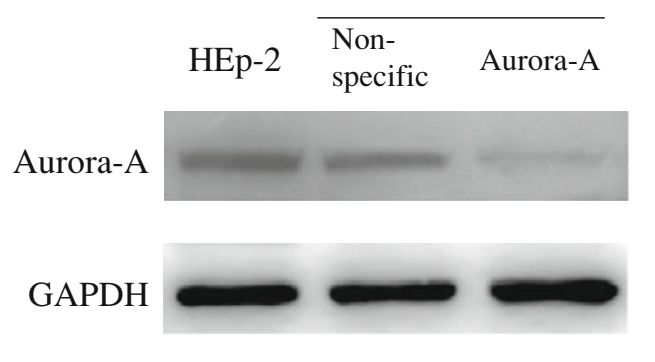

B

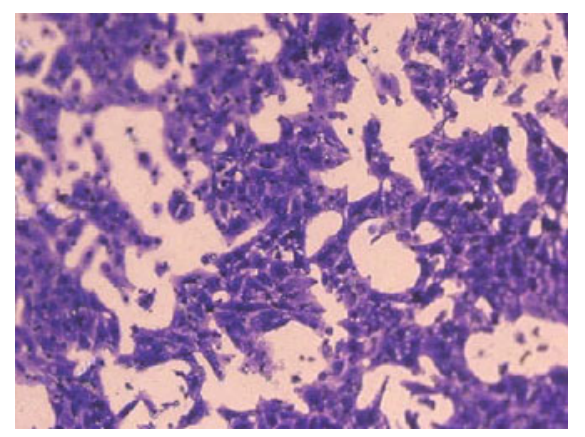

D

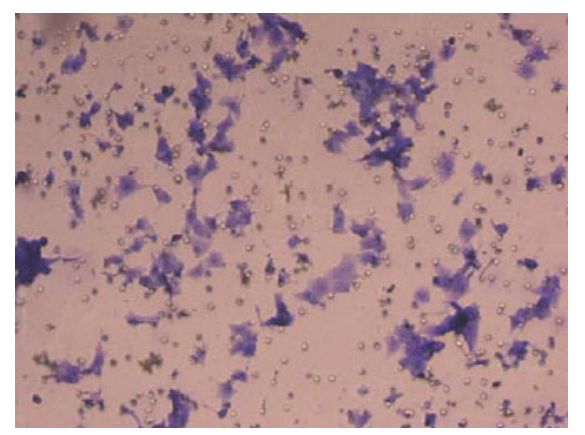

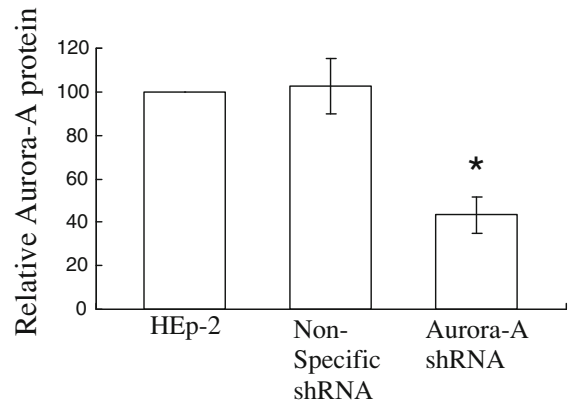

C

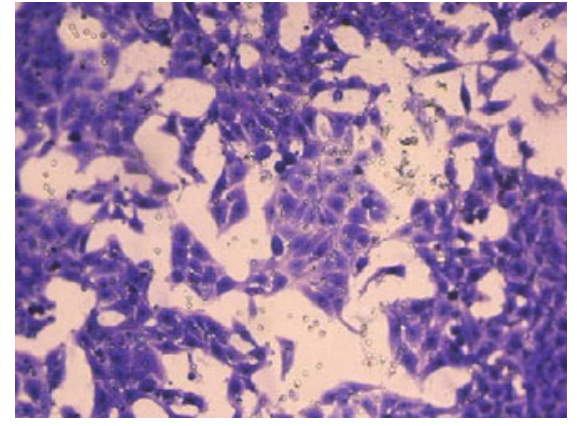

E

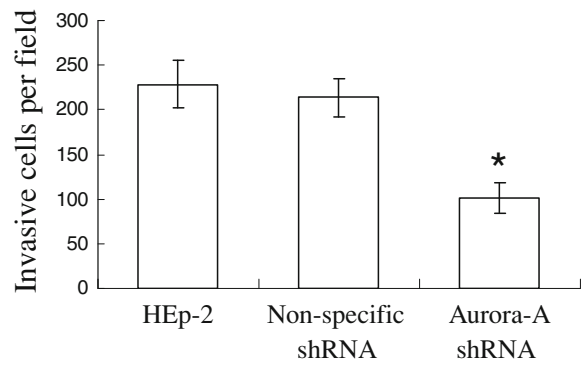

instability events, i.e., anaphase bridge and multipolar mitoses, were measured in anaphase and metaphase HEp-2 cells. The abnormal chromosome segregation was high in HEp-2 cells and remarkably reduced in HEp-2 cells with suppression of Aurora-A. One possible interpretation might be that the fidelity of chromosome segregation is assured in the circumstances of $\mathrm{G} 2 / \mathrm{M}$ checkpoint restoration by Aurora-A suppression. The mutated aneuploid cells were eliminated to the cell cycle. Consequently, the chromosomal instability is prevented. On the contrary, Aurora-A overexpression induces a high level of chromosomal instability that favors the genomic alteration and tumor progression. Thus, our results may shed some light on the mechanism by which overexpression of Aurora-A contributes to tumor progression in laryngeal cancer.

We observed that upregulation of Aurora-A is closely related to regional lymph node metastasis and tumor stage in clinical data. To further clarify whether Aurora-A contributes to the ability of cell invasion, disruption of endogenous Aurora-A protein through RNA interference technique was used in HEp-2 cells. Trans-well assay showed substantially suppression of cell invasion in Aurora-A depleted HEp-2 cells. On the other hand since invasion of tumor cells are essential prerequisites for the metastatic process, invasion and metastasis of tumor to neighboring organs is a main cause of the mortality of LSCC patients. Therefore, these results further suggested that overexpression of Aurora-A might play a major role in regional lymph node metastasis and prognosis of patients. Nevertheless, tumor metastasis is a complex process, involving alterations of cells physical coupling to their microenvironment and activation of extracellular proteases, such as matrix metalloproteinase (MMP), as well as degradation of extracellular matrix [13]. A recent research revealed that MMP-2 and MMP-9 were highly expressed in LSCC patients [14]. However, the signaling pathways interacted between Aurora-A and MMPs in LSCC metastatic mechanisms need further investigation.

The relationship between overexpression of Aurora-A and tumor grade or tumor stage is controversial. Previous 
Fig. 3 The occurrence of chromosomal instability events, measured by anaphase bridges and multipolar mitoses, in HEp2 cell variants. a The occurrence of anaphase bridges or multipolar mitoses in HEp-2 cells transfected with Aurora-A shRNA was significantly lower than that in non-transfected HEp-2. $* P<0.05$. $\mathbf{b}$ and c Representative pictures of anaphase bridge in anaphase cell (×1000). d Representative picture of a multipolar mitotic cell $(\times 1000)$

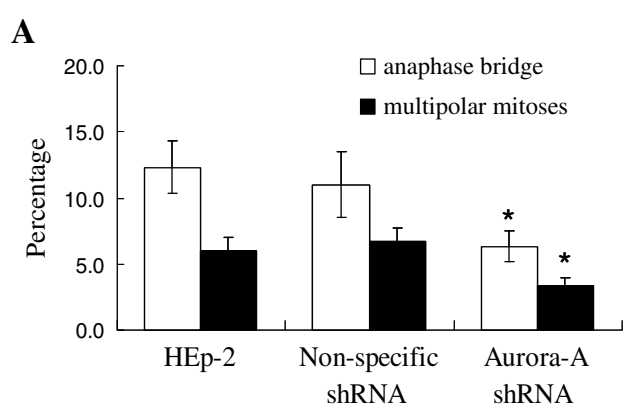

B

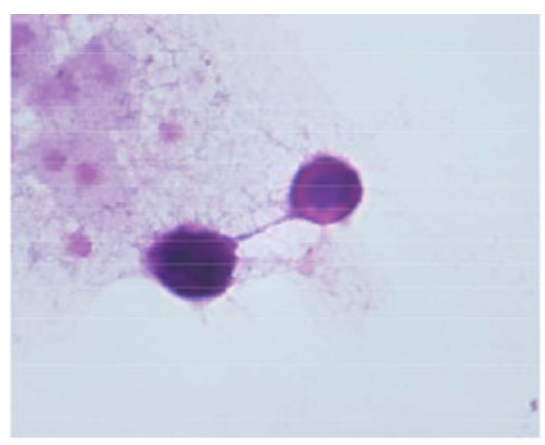

C

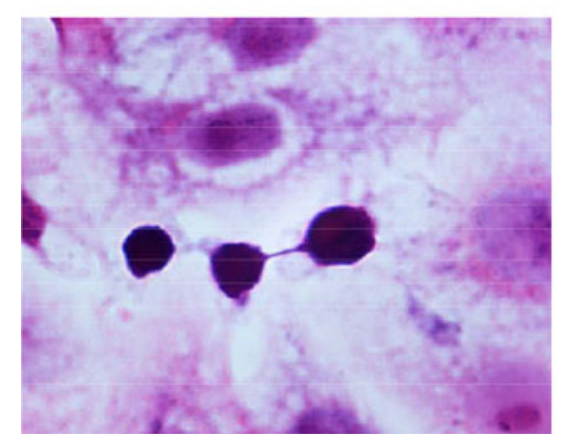

D

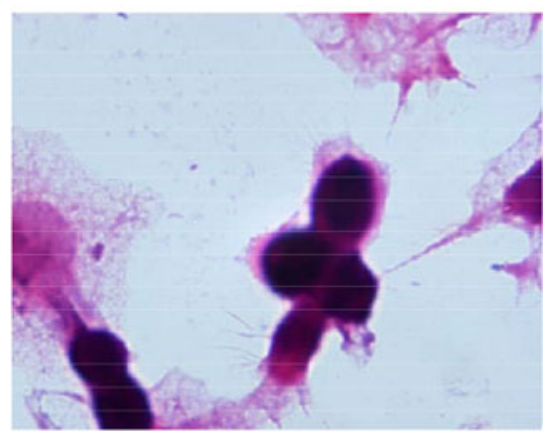

study argued that activation or overexpression of Aurora-A protein was related to low grade and early stage of gastric cancer and ovarian cancer [15, 16]. Moreover, Aurora-A is preferentially expressed in less invasive tumors and declines once a tumor becomes invasive. Other studies showed that elevated expression of Aurora-A was strongly associated with high histological grade and late stage in skin and hepatocellular tumor [10, 17]. In the present study, we observed that upregulation of Aurora-A mRNA was statistically correlated with clinical stage III/IV of LSCC. Aurora-A protein was also overexpressed in patients of tumor stage III/IV, although there was no statistic significance between early stage and late stage tumors. Clinically, when a LSCC patient has regional lymph node metastasis, corresponding to N1-N3, the tumor was classified as stage III or stage IV. Therefore, besides the role of Aurora-A in lymph node metastasis, we supported the view that upregulation of Aurora-A may also be associated with the development of LSCC. This raised the possibility that Aurora-A kinase inhibition may provide for a new approach for the treatment of laryngeal cancer. Future studies are needed to investigate the tumorigenicity of HEp- 2 cells by RNA interference-mediated knockdown of Aurora-A alone or in combination with chemotherapeutic agents either in vitro or in vivo.

In conclusion, our findings suggest that expression of Aurora-A is elevated in human LSCC and is associated with regional lymph metastasis and late stage of tumor. Suppression of Aurora-A reduces invasion ability and chromosomal instability in HEp-2 cells. Thus, overexpression of Aurora-A may contribute to LSCC carcinogenesis and regional metastasis, and could be a potential molecular target for the gene therapy of LSCC.

Acknowledgments This work was supported by the Shanghai Science and Technology Committee (09411950600) and the Chinese National Natural Science Foundation (30670939).

Conflict of interest The authors declared that they have no conflict of interest.

Open Access This article is distributed under the terms of the Creative Commons Attribution Noncommercial License which permits any noncommercial use, distribution, and reproduction in any medium, provided the original author(s) and source are credited.

\section{References}

1. Hunter KD, Parkinson EK, Harrison PR (2005) Profiling early head and neck cancer. Nat Rev Cancer 5:127-135

2. Zhou H, Kuang J, Zhong L et al (1998) Tumour amplified kinase STK15/BTAK induces centrosome amplification, aneuploidy and transformation. Nat Genet 20:189-193

3. Lassmann S, Shen Y, Jutting U et al (2007) Predictive value of Aurora-A/STK15 expression for late stage epithelial ovarian cancer patients treated by adjuvant chemotherapy. Clin Cancer Res 13:4083-4091

4. Baba Y, Nosho K, Shima K et al (2009) Aurora-A expression is independently associated with chromosomal instability in colorectal cancer. Neoplasia 11:418-425

5. Deschler DG, Day T (2008) Pocket guide to neck dissection classification and TNM staging of head and neck cancer, 3rd edn. American Academy of Otolaryngology-Head and Neck Surgery Foundation, Inc, pp 17-19

6. Meraldi P, Honda R, Nigg EA (2002) Aurora-A overexpression reveals tetraploidization as a major route to centrosome amplification in p53-/- cells. EMBO J 21:483-492 
7. Macurek L, Lindqvist A, Lim D et al (2008) Polo-like kinase-1 is activated by aurora A to promote checkpoint recovery. Nature 455:119-123

8. Pascreau G, Eckerdt F, Lewellyn AL et al (2009) Phosphorylation of p53 is regulated by TPX2-Aurora A in xenopus oocytes. J Biol Chem 284:5497-5505

9. Yang G, Chang B, Yang F et al (2010) Aurora kinase A promotes ovarian tumorigenesis through dysregulation of the cell cycle and suppression of BRCA2. Clin Cancer Res 16:3171-3181

10. Jeng YM, Peng SY, Lin CY et al (2004) Overexpression and amplification of Aurora-A in hepatocellular carcinoma. Clin Cancer Res 10:2065-2071

11. Kitajima S, Kudo Y, Ogawa I et al (2007) Constitutive phosphorylation of aurora-A on ser51 induces its stabilization and consequent overexpression in cancer. PLoS One 2:e944

12. Gisselsson D, Jonson T, Yu C et al (2002) Centrosomal abnormalities, multipolar mitoses, and chromosomal instability in head and neck tumours with dysfunctional telomeres. Br J Cancer 87:202-207

13. Hanahan D, Weinberg RA (2000) The hallmarks of cancer. Cell 100:57-70

14. Uloza V, Liutkevicius V, Pangonyte D et al (2011) Expression of matrix metalloproteinases (MMP-2 and MMP-9) in recurrent respiratory papillomas and laryngeal carcinoma: clinical and morphological parallels. Eur Arch Otorhinolaryngol 268(6):871-878

15. Gritsko TM, Coppola D, Paciga JE et al (2003) Activation and overexpression of centrosome kinase BTAK/Aurora-A in human ovarian cancer. Clin Cancer Res 9:1420-1426

16. Kamada K, Yamada Y, Hirao T et al (2004) Amplification/overexpression of Aurora-A in human gastric carcinoma: potential role in differentiated type gastric carcinogenesis. Oncol Rep 12:593-599

17. Torchia EC, Chen Y, Sheng H et al (2009) A genetic variant of Aurora kinase A promotes genomic instability leading to highly malignant skin tumors. Cancer Res 69:7207-7215 